Pacific Journal of Mathematics

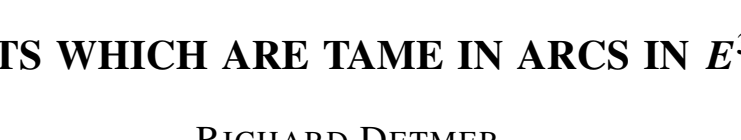




\title{
SETS WHICH ARE TAME IN ARCS IN $E^{3}$
}

\author{
Richard C. Detmer
}

\begin{abstract}
Results of McMillan and Cannon may be combined to give an algebraic condition which is sufficient to show that an arc topologically embedded in $E^{3}$ is tame in $E^{3}$. The main theorem of this paper gives an essentially algebraic condition involving an arc embedded in $E^{3}$ and a compact subset of that arc which is sufficient to show that the arc may be approximated arbitrarily closely without moving the subset, to obtain a tame arc.
\end{abstract}

1. Preliminaries. The usual Euclidean distance function will be denoted by $d$. An open neighborhood having radius $r$ about a set $S$ will by denoted by $N(S, r)$. An $r$-set will be a set having diameter less than $r$.

1.1. Definition. Suppose that $X$ is a compact subset of a finite complex $K$ which is topologically embedded in $E^{3}$. Then $X$ is said to be tame in $K$ iff given $r>0$ there is a homeomorphism $h: K \rightarrow E^{3}$ such that

(1) $d(x, h(x))<r$ for each $x$ in $K$,

(2) $h(x)=x$ for each $x$ in $X$, and

(3) $h(K)$ is tame.

1.2. Definition. Suppose that $X$ is a compact subset of an arc $A$ which is topologically embedded in $E^{3}$. Then $X$ is said to be untangled iff for each $r>0$, there is an $s>0$ such that if $J$ is a loop in $E^{3}-X$ which bounds (homologically) on an $s$-set in $E^{3}-X$, then $J$ shrinks (homotopically) on an $r$-set in $E^{3}-X$.

McMillan [3] has noted that an arc is untangled iff it has free local fundamental groups (1-FLG) at each of its points. He also proved that an arc which has 1-FLG at each point is tame if each of its subarcs pierces a disk. Cannon [2, Theorem 3.16] has shown that an arc which has 1-FLG at each point does pierce a disk. Hence, an arc which is untangled is tame.

1.3. Notation. For the remainder of this paper $A$ will denote an arc topologically embedded in $E^{3}$ and $X$ will denote a compact subset of $A$ which is untangled. The arc $A$ will be assumed to have a fixed order, compatible with, and inducing, the given topology on $A$.

1.4. Definition. Let $Y$ be a subset of $A$. An indexed collection $C_{1}, \cdots, C_{n}$ of disjoint connected subsets of $E^{3}$ is said to be ordered 
with respect to $Y$ iff, in the order on $A$, each point of $C_{i} \cap Y$ precedes each point of $C_{i+1} \cap Y . \quad(i=1, \cdots, n-1$.

The lemma below gives a way to separate components of $X$ by open sets in $E^{3}$ which are, roughly speaking, not much larger than the components.

1.5. Separation Lemma. Suppose that $s$ is a positive number. Then there is a finite cover $C_{1}, \cdots, C_{n}$ of $X$ by connected open subsets of $E^{3}$ with disjoint, polyhedral closures such that:

(1) $C_{1}, \cdots, C_{n}$ is ordered with respect to $X$, and

(2) For each $i$, there is a component $X_{i}$ of $X$ in $C_{i}$ such that $C_{i} \subset N\left(X_{i}, s\right)$.

Proof. The lemma follows easily from the fact that $X$ is a compact set.

2. Cellularity lemmas. In this section it is shown that if $X$ is untangled, then each component $K$ of $X$ can be enclosed in a polyhedral ball which is "close" to $K$ and which has boundary missing $X$. The proof falls naturally into two cases, depending on whether or not $K$ is a nondegenerate component of $X$. The two cases are handled in 2.1 and 2.4 respectively. These results are referred to as cellularity lemmas.

2.1. Cellularity Lemma for Nondegenerate Components. Suppose that $e$ is a positive number and that $K$ is a nondegenerate component of $X$. Then there is a polyhedral ball $B$ such that $K$ is contained in $B, \mathrm{Bd} B$ does not intersect $X$, and $B \subset N(K, e)$.

Proof. By the results of McMillan [3] and Cannon [2], $K$ is a tame arc. Therefore, there is a 3-cell $C \subset N(K, e)$ which contains $K-\mathrm{Bd} K$ in its interior, which does not intersect $A-K$, and which has boundary which is polyhedral modulo $K$.

In view of Dehn's lemma [5], it suffices to prove the fact stated below. Indeed, the fact may be used in conjunction with Dehn's lemma to alter $\mathrm{Bd} C$ slightly near $K \cap \mathrm{Bd} C$ and in $N(K, e)$ so as to miss $X$. The adjusted 2-sphere will then bound a ball $B$ satisfying the requirements of the lemma.

Fact. Suppose that $J$ is a simple closed curve in $\mathrm{Bd} C$ which separates the endpoints of $K$ from each other in $\mathrm{Bd} C$, and suppose that $J$ bounds a disk $D$ in $\mathrm{Bd} C$ of diameter less than some given positive number $q$. Then $J$ bounds a singular disk $D^{\prime}$ in $E^{3}-X$ of diameter less than $q$. 
The fact is proved as follows. Let $r=q-\operatorname{diam} D$. Choose $s>0$ so small that loops which bound on $s$-sets in $E^{3}-X$ shrink on $r$-sets in $E^{3}-X$. Pick a 3-cell $T$ of diameter less than $s$ such that $\mathrm{Bd} T$ separates the endpoints of $K$ in $E^{3}$ and $(\mathrm{Bd} T) \cap(\mathrm{Bd} C)$ is a simple closed curve in Int $D$. Let $E$ denote the disk $C \cap \mathrm{Bd} T$. Use the separation lemma 1.5 to cover components of $X$ which intersect $(\mathrm{Bd} T)-E$ by a finite collection of disjoint open sets whose polyhedral closures miss $E \cup K$. Let $W$ be the union of these sets and assume that $\mathrm{Bd} W$ is in general position with respect to $\mathrm{Bd} T$. Because Cl $W$ does not intersect $K \cup E, \mathrm{Bd} E$ bounds homologically on the $s$-set $\mathrm{Bd}(T-W)-\operatorname{Int} E$ in $E^{3}-X$ and therefore bounds a singular $r$-disk $F$ in $E^{3}-X$. The set $(D-K) \cup F$, which lies in $E^{3}-X$, contains a singular disk $D^{\prime}$ of diameter less than $q$ which is bounded by $J$. This establishes the fact and completes the proof of the lemma.

If, in the proof of $2.1, C$ is first partitioned by means of disjoint spanning disks $D_{1}$ and $D_{2}$ into three 3-cells - a central 3-cell $C_{3}$, whose intersection with $K$ is an arc $K_{3} \subset \operatorname{Int} K$, and end cells $C_{1}$ and $C_{2}$ whose intersections with $K$ are the closed components $K_{1}$ and $K_{2}$ of $K-K_{3}$ and $\mathrm{Bd} C$ is adjusted only very near $\left(\mathrm{Bd} C_{1}-D_{1}\right) \cap K$ and $\left(\mathrm{Bd} C_{2}-\right.$ $\left.D_{2}\right) \cap K$ in constructing $\mathrm{Bd} B$, then the following is evident.

2.2. ADDENDUM. The ball $B$ in 2.1 may be chosen in such a manner that it can be partitioned by disjoint spanning disks $D_{1}$ and $D_{2}$ into 3-cells $B_{1}, B_{2}$, and $B_{3}\left(B_{i} \cap B_{3}=D_{i}\right.$ for $i=1$,2) satisfying to $D_{2}$,

(1) $B_{3} \cap A$ is a subarc of Int $K$ which spans the cell $B_{3}$ from $D_{1}$

(2) the diameter of $B_{i}$ is less than $e(i=1,2)$, and

(3) $B_{i} \cap A$ lies in an $e$-arc on $A(i=1,2)$ with one endpoint of this $e$-arc missing $X$ (unless $B_{i}$ contains an endpoint of $A$ ).

The next lemma is well-known.

2.3. Lemma. Suppose that $J$ is a simple closed curve in $E^{3}$ which bounds an orientable surface $T$ of diameter less than $r$, and suppose that $L$ is an arc of diameter less than $s$ which misses $J$. Then $J$ bounds a surface of diameter less than $r+s$ in $E^{3}-L$ and this surface may be chosen in an arbitrarily small neighborhood of $T \cup L$.

2.4. Cellularity Lemma for Degenerate Components. Suppose that $s$ is a positive number and that $\{p\}$ is a degenerate component of $X$. Then there is a polyhedral 3-cell $B$ such that $p$ lies in $B$, $\mathrm{Bd} B \cap X=\varnothing$, and $B \subset N(p, s)$.

Proof. As a first approximation to the desired 3-cell, let $B^{\prime}=$ $\mathrm{Cl}(N(p, r))$ where $0<r<s / 2$ and $r$ is so small that $B^{\prime}$ intersects no 
component of $X$ with diameter as large as $s / 4$. Choose $q>0$ so small that loops which bound on $q$-sets in $E^{3}-X$ shrink on $r / 2$-sets in $E^{3}-X$.

Choose a collection $D_{1}, \cdots, D_{n}$ of small disjoint polyhedral disks on $\mathrm{Bd} B^{\prime}$ with boundaries missing $X$ and a collection $S_{1}, \cdots, S_{m}$ of small polyhedral spheres in $E^{3}-X$ such that $X \cap \mathrm{Bd} B^{\prime}$ is contained in $D_{1} \cup \cdots \cup D_{n} \cup\left(\operatorname{Int} S_{1} \cap \mathrm{Bd} B^{\prime}\right) \cup \cdots \cup\left(\operatorname{Int} S_{m} \cap \mathrm{Bd} B^{\prime}\right)$. Specifically, select for each degenerate component $\{x\}$ of $X$ which lies on $\mathrm{Bd} B^{\prime}$ a disk $D_{x} \subset \mathrm{Bd} B^{\prime}$ with $\mathrm{Bd} D_{x} \cap X=\varnothing$ and so small that diam $D_{x}<q / 2$ and $D_{x} \cap A$ lies on a subarc of $A$ with diameter less than $q / 2$ which has endpoints in $A-X$. Select a sphere for each nondegenerate component of $X$ which intersects $\mathrm{Bd} B^{\prime}$ using the cellularity lemma for nondegenerate components, 2.1, with $s / 4$ as the value of $e$ in that lemma. A finite collection of these disks and spheres satisfies the conditions above except for the requirement that the disks be disjoint. Since their boundaries miss $X$, the disks may be made disjoint by simply putting their boundaries in general position and cutting them apart.

Application of Lemma 2.3 shows that, for each $i, \mathrm{Bd} D_{i}$ bounds on a $q$-set in $E^{3}-X$, and hence shrinks on an $r / 2$-set in $E^{3}-X$. Because of this, if Int $D_{i}$ is thrown away and $\mathrm{Bd} D_{i}$ shrunk, a singular 2 -sphere $R_{0}$ may be produced which intersects $X$ in "fewer" places than did $\mathrm{Bd} B^{\prime}$ and which is essential in $E^{3}-\{p\}$ since it is homotopic to $\mathrm{Bd} B^{\prime}$ in $E^{3}-N(p, r / 2)$. If any of the spheres $S_{i}$ contains $p$, the proof is finished since each $S_{i}$ has diameter less than $s$. Otherwise, for each $i$ in turn, choose a point $x_{i}$ in Int $S_{i}-R_{i-1}$ and project "radially" the part of $R_{i-1}$ in Int $S_{i}$ into $S_{i}$ to form a singular sphere $R_{i}$. The final result is a singular sphere $R_{m}$ in $E^{3}-X$, essential in $E^{3}-\{p\}$, and lying in $N(p, s)$. Using a strong form of the sphere theorem (which is implicit in the original proof in [5] and is stated explicitly in [6]), there is a nonsingular, polyhedral sphere with the same properties. Taking $B$ to be the closure of the interior of this sphere completes the proof.

3. The following theorem is the main result of this paper. It says that if a compact subset of an arc is untangled, then it is tame in that arc.

Theorem 3.1. Suppose that $X$ is a compact subset of an arc $A$ which is topologically embedded in $E^{3}$, and that for each positive number $r$, there is a positive number $s$ such that if $J$ is a loop in $E^{3}-X$ which bounds on an s-set missing $X$, then $J$ shinks on an $r$-set missing $X$. Then for each positive number $q$ there is a homeomorphism $f: A \rightarrow E^{3}$ such that

(1) $f(x)=x$ for each $x$ in $X$, 
(2) $d(x, f(x))<q$ for each $x$ in $A$, and

(3) $f(A)$ is tame.

Proof. The idea of the proof is as follows. Construct a homeomorphism $h: E^{3} \rightarrow E^{3}$ such that the restriction of $h$ to $X$ takes $X$ in an order preserving fashion into the $x$-axis. Define $f(A)=h^{-1}(I)$ where $I$ is a suitably chosen subinterval of the $x$-axis. Care must be taken in the construction of $h$ in order that $A$ and $f(A)$ be close homeomorphically. The details of this process are described below.

It may be assumed that $A$ is locally polyhedral modulo $X$. Use the cellularity lemma for nondegenerate components, 2.1 , to construct a collection $B_{1}^{\prime}, \cdots, B_{n}^{\prime}$ of disjoint polyhedral 3-cells with boundaries in $E^{3}-X$, one for each component of $X$ with diameter as large as $q / 12$. Choose $e$ in that lemma and its addendum, 2.2, less than $q / 6$, and so small that the collection of cells is ordered with respect to $X$ (although it need not cover $X$ ).

The set $X^{\prime}=X-\bigcup B_{i}^{\prime}$ is a compact subset of $A$ with no component having diameter as large as $q / 12 ; X^{\prime}$ is untangled. "Small" 3-cells covering $X^{\prime}$ are now constructed. By an appropriate choice of $s$ in the separation lemma, 1.5, a cover $C_{1}, \cdots, C_{m}$ of $X^{\prime}$ may be obtained, the closures of whose elements miss $\cup B_{i}^{\prime}$, have diameters less than $q / 6$, and are ordered with respect to $X$. A suitable choice of $s$ also guarantees that each $C_{i}$ intersects at most one of the $q / 6$ arcs associated (by the Addendum 2.2) with any $B_{j}^{\prime}$, and that $C_{i} \cap A$ lies in a $q / 6$-arc on $A$.

Each $C_{i}$ may be changed to a ball, using the two cellularity lemmas to cover each component of $X$ inside $C_{i}$ by a polyhedral ball, picking a finite subcover of these, and following the methods of Bing [1] to cut apart and reconnect these balls to form a single ball containing $X \cap C_{i}$ in its interior. Retain the notation $C_{i}$ for such a polyhedral ball.

Modify each $B_{j}^{\prime}(j=1, \cdots, n)$ as follows. The set $B_{j}^{\prime} \cap C \mathrm{l}\left(A-B_{j}^{\prime}\right)$ is contained in (at most) two $q / 6$-arcs $A_{1}$ and $A_{2}$ on $A$, and the intersection of each of these with $B_{j}^{\prime}$ lies in a $q / 6$-cell (by the addendum). If $A_{i}$ intersects $X-B_{j}$, then $A_{i} \cap\left(X-B_{j}\right)$ is contained in some of the $C_{i}$ 's. Connect these $C_{i}$ 's to the $q / 6$-cells using disjoint $q / 6$-cells, so that the result is two $5 q / 6$-cells, each of which intersects one of $A_{1}$ or $A_{2}$ and no other point of $A-B_{j}^{\prime}$.

Let $B_{1}, \cdots, B_{n}, B_{n+1}, \cdots, B_{k}$ be the modified $B_{j}$ 's $(j=1, \cdots, n)$ plus the $C_{j}$ 's not used in the modifications. Assume that the indices are arranged so that this collection is ordered with respect to $X$.

Figure 1 illustrates the situation at this point of the proof.

The purpose in constructing $B_{1}, \cdots, B_{n}$ so carefully is to make it possible to change $A$ homeomorphically by moving only points of 


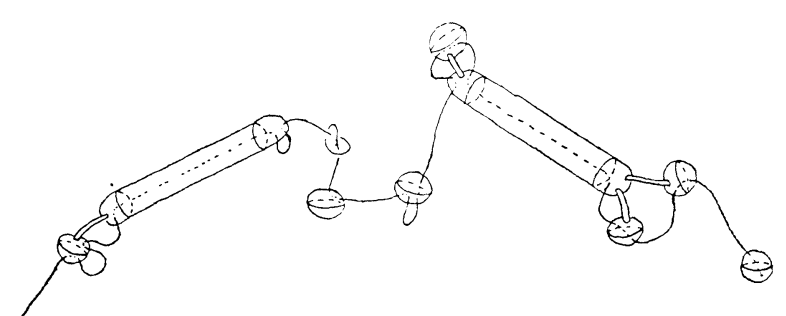

FIGURE 1

$A$ which are near small components of $X$ and near the endpoints of large components of $X$. Define a homeomorphism $f^{\prime}$ on $A$ to move some subarcs of the $q / 6$-arcs associated with $B_{1}, \cdots, B_{n}$ into the $5 q / 6$ cells at the "ends" of these "large" balls, and to move some subarcs of the $q / 6$-arcs associated with $B_{n+1}, \cdots, B_{k}$ into these balls; do this so that $f^{\prime}(A)$ intersects each $\mathrm{Bd} B_{j}$ in at most two points (and only one point if $B_{j}$ (contains an endpoint of $A$.)

The first approximation $h_{1}$ to the homeomorphism $h$ taking $X$ into the $x$-axis is defined to take $f^{\prime}(A)-\bigcup B_{i}$ into the $x$-axis, to take each $B_{i}$ to a small neighborhood of an arc on the $x$-axis, and to take each component of $X$ with diameter as large as $q / 12$ into the $x$-axis, everything with order preserved. Subsequent approximations to $h$ will be the identity outside the images under $h_{1}$ of the $5 q / 6$-cells associated with $B_{1}, \cdots, B_{n}$ and of $B_{n+1}, \cdots, B_{k}$, and this will ensure that conclusion (2) of the theorem is true.

Approximations to $h$ are now obtained sequentially. At the second stage of the construction, a new collection of balls is chosen, inside the fiast, closer to $X$, and separating the components of $X$ with diameters as large as $q / 24$. A homeomorphism $h_{2}$ of $E^{3}$ onto itself is obtained which is the identity on each of the $h_{1}$-images of the components of $X$ which have diameter as large as $q / 12$. This homeomorphism maps $h_{1}$-images of the new balls to neighborhoods of arcs on the $x$-axis, and $h_{2} h_{1}$ also sends components of $X$ with diameter as large as $q / 24$ into the $x$-axis, preserving order of cells.

Continue in this manner, choosing the balls so small that the sequence $h_{1}, h_{2} h_{1}, h_{3} h_{2} h_{1}, \cdots$ of homeomorphisms converges to a homeomorphism $h$. Let $I$ be the smallest subarc of the $x$-axis which contains $h\left(f^{\prime}(A)\right) \cap\left(x\right.$-axis). The arc $h^{-1}(I)$ differs from $f^{\prime}(A)$ only in the end cells of $B_{1}, \cdots, B_{n}$ and in $B_{n+1}, \cdots, B_{k}$ and so a homeomorphism $f: A \rightarrow E^{3}$ may be defined so that $f(A)=h^{-1}(I)$ and $d(x, f(x))<q$ for each $x$ in $A$. The homeomorphism $f$ may also be chosen to fix points of $X$ since the restriction of $h$ to $X$ is a homeomorphism of $X$ into the $x$-axis. This completes the proof of the theorem.

There is also a relative version of 3.1 :

Theorem 3.2. Suppose that $X$ is a compact subset of an arc $A$ 
which is topologically embedded in $E^{3}$ and that $X$ is untangled. Suppose that $g: A \rightarrow[0, \infty)$ is a continuous function so that $g^{-1}(0) \cap$ $X=\varnothing$. Then there is a homeomorphism $f: A \rightarrow E^{3}$ such that

(1) $f(x)=x$ for each $x$ in $X$,

(2) $d(x, f(x))<g(x)$ for each $x$ in $A$, and

(3) $f(A)$ is locally tame modulo the set $g^{-1}(0)$.

Proof. The proof is almost exactly the same as for 3.1, except for beginning with an approximation to $A$ which is locally polyhedral modulo $X \cup g^{-1}(0)$ and which is homeomorphically within $g$ of $A$. This new arc is then modified on subarcs close to $X$ in the same way as in the proof of 3.1 .

4. Theorem 3.1 may be combined with a characterization of subsets of arcs due to R. L. Moore [4, Theorem 135] to yield a characterization of subsets of tame arcs in $E^{3}$. Moore's theorem is proved for a space satisfying his axioms $0-5$ and $E^{3}$ does not satisfy axiom 4. However the proof is not difficult in this case.

TheOREM (R. L. Moore) 4.1. In order that the compact point set $M$ in $E^{3}$ be a subset of an arc it is necessary and sufficient that every closed and connected subset of $M$ be either a degenerate point set or an arc $t$ such that no point of $t$, except for its endpoints is a limit point of $M-t$.

4.2. Characterization of Subsets of Tame Arcs in $E^{3}$. Suppose that $X$ is a compact subset of $E^{3}$. Then $X$ is a subset of a tame arc in $E^{3}$ if and only if each component of $X$ is a point or an arc $t$ such that no point of $t$, except possibly for an endpoint, is a limit point of $X-t$, and for each positive number $r$, there is a positive number $s$ such that each loop which bounds on a $s$-set in $E^{3}-X$ shrinks on an $r$-set in $E^{3}-X$.

Proof. Sufficiency follows from 3.1 and 4.1. Necessity is obvious.

\section{REFERENCES}

1. R. H. Bing, Tame Cantor sets in $E^{3}$, Pacific J. Math., 11 (1961), 435-446.

2. J. W. Cannon, ULC properties in neighborhoods of embedded surfaces and curves in $E^{3}$, Canad. J. Math., 25 (1973), 31-73.

3. D. R. McMillan, Local properties of the embedding of a graph in a three-manifold, Canad. J. Math., 18 (1966), 517-528.

4. R. L. Moore, Foundations of Point Set Theory, rev. ed., Amer. Math. Soc. Colloq. Pub., vol. 13, Amer. Math. Soc., 1962.

5. C. D. Papakyriakopolous, On Dehn's lemma and the asphericity of knots, Ann. of 
Math., (2) 66 (1957), 1-26.

6. J. H. C. Whitehead, On 2-spheres in 3-manifolds, Bull. Amer. Math. Soc., 64 (1958), 161-166.

Received December 12, 1972. This paper formed part of the author's doctoral thesis at the University of Wisconsin, Madison, under the direction of Professor J. W. Cannon. University of Tennessee at Chattanooga 


\section{PACIFIC JOURNAL OF MATHEMATICS}

\section{EDITORS}

RICHARD ARENS (Managing Editor)

University of California

Los Angeles, California 90024

R. A. BeAumont

University of Washington

Seattle, Washington 98105
J. DugundJI*

Department of Mathematics

University of Southern California

Los Angeles, California 90007

D. Gilbarg and J. Milgram

Stanford University

Stanford, California 94305

\section{ASSOCIATE EDITORS}

E. F. BECKENBACH

B. H. NEUMANN

F. WOLF

K. YoSHIDA

\section{SUPPORTING INSTITUTIONS}

UNIVERSITY OF BRITISH COLUMBIA
CALIFORNIA INSTITUTE OF TECHNOLOGY
UNIVERSITY OF CALIFORNIA
MONTANA STATE UNIVERSITY
UNIVERSITY OF NEVADA
NEW MEXICO STATE UNIVERSITY
OREGON STATE UNIVERSITY
UNIVERSITY OF OREGON
OSAKA UNIVERSITY

UNIVERSITY OF BRITISH COLUMBIA CALIFORNIA INSTITUTE OF TECHNOLOGY UNIVERSITY OF CALIFORNIA MONTANA STATE UNIVERSITY NEW MEXICO STATE UNIVERSITY UNIVERSITY OF OREGON OSAKA UNIVERSITY
UNIVERSITY OF SOUTHERN CALIFORNIA STANFORD UNIVERSITY UNIVERSITY OF TOKYO UNIVERSITY OF UTAH WASHINGTON STATE UNIVERSITY UNIVERSITY OF WASHINGTON AMERICAN MATHEMATICAL SOCIETY NAVAL WEAPONS CENTER

* C. R. DePrima California Institute of Technology, Pasadena, CA 91109, will replace J. Dugundji until August 1974. 


\section{Pacific Journal of Mathematics}

\section{Vol. 51, No. $1 \quad$ November, 1974}

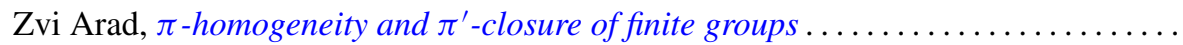

Ivan Baggs, A connected Hausdorff space which is not contained in a maximal

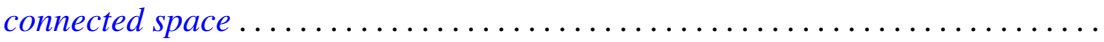

Eric Bedford, The Dirichlet problem for some overdetermined systems on the unit ball in $C^{n}$

R. H. Bing, Woodrow Wilson Bledsoe and R. Daniel Mauldin, Sets generated by

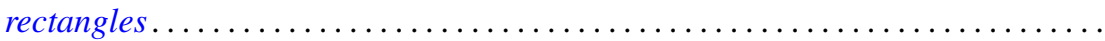

Carlo Cecchini and Alessandro Figà-Talamanca, Projections of uniqueness for

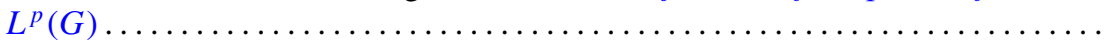

Gokulananda Das and Ram N. Mohapatra, The non absolute Nörlund summability of Fourier series .

Frank Rimi DeMeyer, On separable polynomials over a commutative ring ........ Richard Detmer, Sets which are tame in arcs in $E^{3} \ldots \ldots \ldots \ldots \ldots \ldots \ldots \ldots$

William Erb Dietrich, Ideals in convolution algebras on Abelian groups ..........

Bryce L. Elkins, A Galois theory for linear topological rings .................

William Alan Feldman, A characterization of the topology of compact convergence on $C(X)$.

Hillel Halkin Gershenson, A problem in compact Lie groups and framed cobordism

Samuel R. Gordon, Associators in simple algebras.

Marvin J. Greenberg, Strictly local solutions of Diophantine equations

Jon Craig Helton, Product integrals and inverses in normed rings . . . . . . . . . . . .

Domingo Antonio Herrero, Inner functions under uniform topology . . .

Jerry Alan Johnson, Lipschitz spaces .

Marvin Stanford Keener, Oscillatory solutions and multi-point boundary value

functions for certain nth-order linear ordinary differential equations.

John Cronan Kieffer, A simple proof of the Moy-Perez generalization of the

Shannon-McMillan theorem .......................

Joong Ho Kim, Power invariant rings

Gangaram S. Ladde and V. Lakshmikantham, On flow-invariant sets .

Roger T. Lewis, Oscillation and nonoscillation criteria for some self-adjoint even

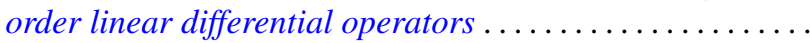

Jürg Thomas Marti, On the existence of support points of solid convex sets ..

John Rowlay Martin, Determining knot types from diagrams of knots . .

James Jerome Metzger, Local ideals in a topological algebra of entire functions

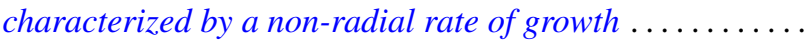

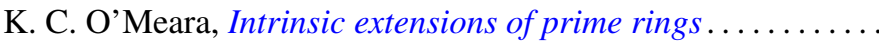

Stanley Poreda, A note on the continuity of best polynomial approximations ..

Robert John Sacker, Asymptotic approach to periodic orbits and local prolongations

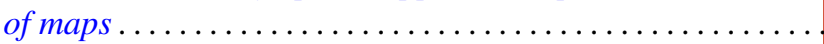

Eric Peter Smith, The Garabedian function of an arbitrary compact set . .

Arne Stray, Pointwise bounded approximation by functions satisfying a side condition

John St. Clair Werth, Jr., Maximal pure subgroups of torsion complete abelian

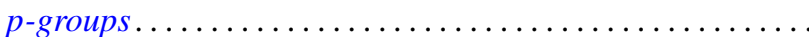

